\title{
Correlation between genotype and the tetrahydrobiopterin-responsive phenotype in Chinese patients with phenylketonuria
}

\author{
Jing Tao ${ }^{1,2}$, Nana Li ${ }^{1,3}$, Haitao Jia ${ }^{4}$, Zhen Liu' ${ }^{1,3}$, Xiaohong Li ${ }^{1,3}$, Jiaping Song ${ }^{4}$, Ying Deng ${ }^{1,3}$, Xi Jin $^{1,3}$ and Jun Zhu ${ }^{1}$
}

\begin{abstract}
BACKGROUND: A growing body of research has suggested that tetrahydrobiopterin $(\mathrm{BH} 4)$ responsive phenotype can be predicted by the phenylalanine hydroxylase $(P A H)$ genotype in patients with phenylketonuria (PKU), but data concerning the association between genotype and $\mathrm{BH} 4$ responsiveness are scarce in China.
\end{abstract}

METHODS: A total of 165 PKU patients from China who had undergone a 24-h loading test with $\mathrm{BH} 4$ administration were recruited. Genotyping was performed by the next-generation sequencing (NGS) technique. Using the predicted residual PAH activity, we analyzed the association between genotype and BH4-responsiveness.

RESULTS: Among the 165 patients, 40 patients (24.24\%) responded to $\mathrm{BH} 4$. A total of 74 distinct mutations were observed, including 13 novel mutations. The mutation p.R241C was most frequently associated with response. Two known mutations (p.A322T and p.Q419R) and two novel mutations (p.L98V and IVS3-2A>T) were first reported as responsive to $\mathrm{BH}$. Residual PAH activity of at least $12.5 \%$ was needed for responsive genotypes.

CONCLUSION: Genotype-based predictions of BH4responsiveness are only for selecting potential responders. Accordingly, it is necessary to test potential responders with a long-term $\mathrm{BH} 4$ challenge.

$\mathrm{P}$ henylketonuria (PKU, MIM261600) is an autosomal recessive disorder of amino acid metabolism and is caused by mutations in the phenylalanine hydroxylase $(P A H, \mathrm{EC}$ 1.14.16.1) gene. A mutant $P A H$ gene gives rise to the decreased ability of the PAH enzyme to convert phenylalanine (Phe) to tyrosine (Tyr), which subsequently leads to the accumulation of Phe and its secondary metabolites in the body. A variety of clinical problems (e.g., intellectual impairment, autistic behavior, and seizures) manifest in patients with PKU unless treatment starts soon enough after birth (1). PKU falls into the most common inborn error of amino acid metabolism in China, with an incidence of 1:10,000 (2). So far, early identification followed by effective treatment for PKU patients play key roles in preventing the devastating consequences of PAH deficiency.

$\mathrm{BH} 4$ is the natural cofactor of $\mathrm{PAH}$, and its pharmaceutical formulation (Sapropterin dihydrochloride) is an efficacious treatment with no severe adverse events for a subset of PKU patients (3). BH4 administration focuses not only on stabilizing the blood Phe level within the therapeutic range but also on improving dietary Phe tolerance by enhancing the catabolism of blood Phe (4-6). Not all patients are responsive to BH4 treatment, and most responders have mild biochemical phenotypes $(7,8)$. However, there is no consensus on the gold standard test for ascertaining $\mathrm{BH} 4$ responsiveness in $\mathrm{PKU}$ patients.

Some evidence has indicated that the BH4-responsive genotype is characterized by substantial residual PAH activity (9-11). Moreover, there is a significant difference of $\mathrm{PAH}$ activity between $\mathrm{BH} 4$ responders and nonresponders (12). These may be related to the molecular mechanism behind $\mathrm{BH} 4$ response, including stabilizing the altered conformation of the PAH enzyme and preventing its degradation $(13,14)$. Until now, more than 850 mutations have been reported in the BIOPKU database (University of Zurich, Zurich, Switzerland) (15). Therefore, mutation screening is of value to select potential responders and to exclude nonresponders before $\mathrm{BH} 4$ trial.

Due to the allelic heterogeneity in PKU patients from different geographical areas (16), it is worthwhile to detect mutant $\mathrm{PAH}$ genotypes for $\mathrm{BH} 4$ responsiveness in the Chinese population. Next-generation sequencing (NGS) has emerged as an efficient method for disease diagnosis, with the application of complete genome resequencing, transcriptome sequencing, small RNA sequencing, sequencing of bisulfite-treated DNA and so on (17). Currently, NGS has been performed for the genetic diagnosis of PKU, with increased diagnostic sensitivity and shorter analysis time and lower costs $(18,19)$. This study is using NGS to analyze the $P A H$ gene in a cohort of Chinese PKU patients, with the aim of assessing the association between genotype and $\mathrm{BH} 4$ responsiveness. 
Articles | Tao et al.

Table 1. Spectrum of $P A H$ mutations identified in $165 \mathrm{PKU}$ patients

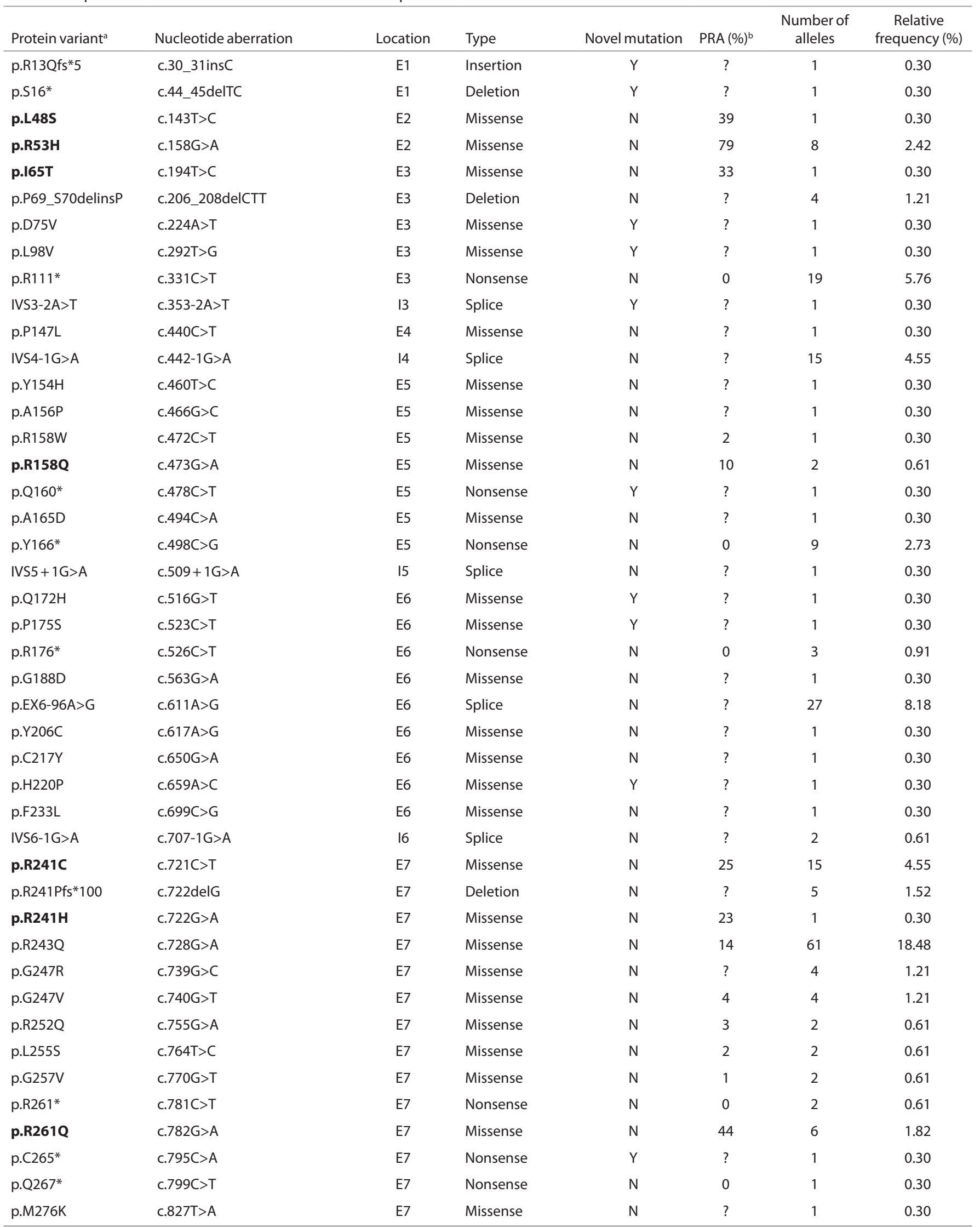

Table 1. Continued on next page 
Table 1. Continued

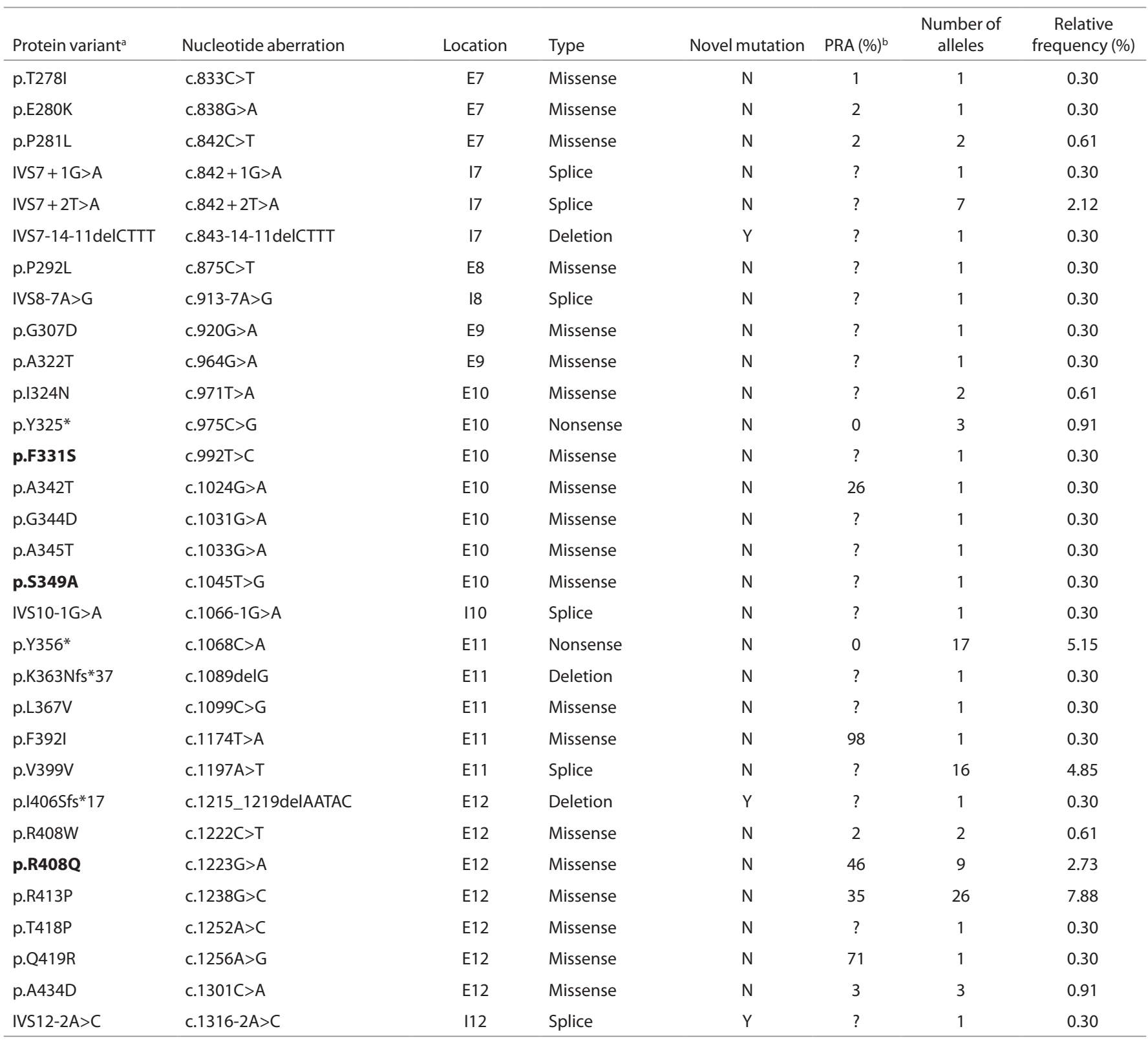

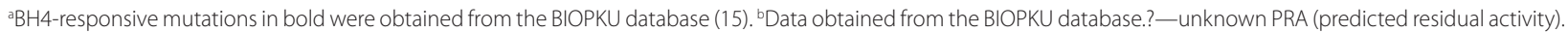

\section{RESULTS}

Patients were classified into three biochemical phenotypes on the basis of the pretreatment blood phenylalanine concentration at the first definite diagnosis: 100 patients $(60.61 \%)$ with classical PKU (cPKU, Phe levels $>20 \mathrm{mg} / \mathrm{dl}$ ), 49 patients $(29.70 \%$ ) with mild PKU (mPKU, Phe levels $10-20 \mathrm{mg} / \mathrm{dl}$ ), and 16 patients $(9.70 \%)$ with mild hyperphenylalaninemia (MHP, Phe levels $<10 \mathrm{mg} / \mathrm{dl})$. Among the 40 patients (24.24\%) responsive to $\mathrm{BH} 4,13$ patients were characterized with mild hyperphenylalaninemia, 23 patients with $\mathrm{mPKU}$, and 4 patients with cPKU.

\section{PAH Mutation Spectrum}

Mutations were identified on both alleles for 153 patients and on only a single allele for 12 patients, corresponding to a diagnostic efficiency of $96.36 \%$ (318/330). Among the 153 patients,
6 patients were carrying more than two mutations. A total of 75 distinct mutations were discovered (Table 1): 48 missense mutations, 11 splicing mutations, 9 nonsense mutations, 6 small deletion mutations, and 1 insertion mutation. These mutations were scattered in all exons and introns, except in introns $1,2,9$, and 11 . Over $65 \%$ of the mutations were localized in exons $7,12,6$, and 11.

Eight mutations (p.R243Q, p.EX6-96A>G, p.R413P, p.R111X, p.Y356X, p.V399V, p.R241C, and IVS4-1G>A), each with a frequency $\geq 3 \%$, accounted for $59.39 \%$ of the alleles. The most prevalent mutation was p.R243Q, with a relative frequency of $18.48 \%$. The frequency for the other seven mutations accounted for $8.18,7.88,5.76,5.15,4.85,4.55$, and $4.55 \%$.

A total of 13 novel mutations (Table 1), each occurring only once, have not been previously reported or listed in the 
PAHdb or BIOPKUdb. These novel mutations were identified in relevant parents. Meanwhile, these mutations were not present in the control group of 200 healthy individuals of Chinese origin and the $>5,000$ individuals sequenced and documented in the Exome Variant Server. Details on the pathologic analysis of five missense mutations are provided in Table 2.

\section{Correlation Between Genotype and BH4 Responsiveness} In patients with a genotype consisting of two mutations. A total of 147 patients carrying two mutations constituted a spectrum of 105 different genotypic combinations (Table 3). The genotypes were divided into homozygous $(n=15)$ and heterozygous $(n=132)$. The heterozygous genotypes were further subdivided into three groups: null+null $(n=25)$, missense+null/functionally heterozygous $(n=74)$, and missense+missense/compound heterozygous $(n=33)$.

By analyzing the homozygous genotypes or functionally heterozygous genotypes, eight mutations were associated with responsiveness, including six known mutations (p.R241C, p.A322T, p.F331S, p.S349A, p.R408Q, and p.Q419R) and two novel mutations (p.L98V and IVS3-2A $>$ T). However, four mutations (p.R53H, p.I65T, p.R158Q, and p. R261Q) previously defined as $\mathrm{BH} 4$ responsive were negative to $\mathrm{BH} 4$.

Three kinds of genotypes (p.[R111*]; EX6-96A>G], p.[R413P]; [Y356*], p.[A434D];[EX6-96A>G]) showed disparate $\mathrm{BH} 4$-responsiveness. A good genotype-phenotype correlation was observed in the remaining genotypic types $(n=$ $102)$, which were unambiguously either responsive $(n=29$, $28.43 \%)$ or unresponsive $(n=73,71.57 \%)$ to BH4. Among these genotypes with the known predicted residual activity (PRA) in both mutations, the lowest residual PAH activity of the responsive genotype was at least $12.5 \%$, and the highest residual $\mathrm{PAH}$ activity of the nonresponsive genotype was up to $46.5 \%$.

As for the data on the BH4-responsiveness, only 6 of the 105 genotypes existed in the BIOPKU database. However, discordant $\mathrm{BH} 4$-responsiveness was observed in the two genotypes (p.[R53H];[R243Q] and p.[I65T];[R243Q]).

Patients with a genotype consisting of more than two mutations. Six patients with three or four mutations were observed (Table 4). Parents studies demonstrated that one missense mutation (p.R53H) and one splicing mutation (IVS7+2T>A) were always found on a common chromosome. However, these patients having the genotype with at least $26.33 \%$ residual PAH activity did not respond to $\mathrm{BH} 4$.

\section{DISCUSSION}

Sapropterin dihydrochloride (BH4 pharmaceutical formulation) has been approved by the Food and Drug Administration in the United States and by the European Medicines Agency in Europe to treat a subset of PKU patients. Molecular analysis of the $P A H$ gene would assist in predicting which patients could benefit from the $\mathrm{BH} 4$ therapeutic approach, which can alleviate the diet burden (20).Thus, the investigation of mutant
Table 2. Pathologic analysis of five novel mutations of the $P A H$ gene

\begin{tabular}{|c|c|c|c|c|}
\hline \multirow[b]{2}{*}{$\begin{array}{l}\text { Protein } \\
\text { variant }\end{array}$} & \multirow[b]{2}{*}{$\begin{array}{l}\text { Nucleotide } \\
\text { aberration }\end{array}$} & \multicolumn{3}{|c|}{ Classification with bioinformatics tools } \\
\hline & & $\begin{array}{l}\text { Polyphen2 } \\
\text { (score) }\end{array}$ & $\begin{array}{l}\text { SIFT Blink } \\
\text { (score) }\end{array}$ & $\begin{array}{l}\text { SNPs3D } \\
\text { (score) }\end{array}$ \\
\hline p.D75V & c. $224 \mathrm{~A}>\mathrm{T}$ & $\begin{array}{l}\text { Benign } \\
(0.373)\end{array}$ & $\begin{array}{l}\text { Tolerated } \\
(0.210)\end{array}$ & $\begin{array}{l}\text { Tolerated } \\
(1.030)\end{array}$ \\
\hline p.L98V & c. $292 \mathrm{~T}>\mathrm{G}$ & $\begin{array}{l}\text { Possibly } \\
\text { damaging (0.532) }\end{array}$ & $\begin{array}{l}\text { Tolerated } \\
(0.380)\end{array}$ & $\begin{array}{l}\text { Tolerated } \\
(0.670)\end{array}$ \\
\hline p.Q172H & c.516G $>\mathrm{T}$ & $\begin{array}{l}\text { Possibly } \\
\text { damaging (0.921) }\end{array}$ & $\begin{array}{l}\text { Damaging } \\
(0.029)\end{array}$ & $\begin{array}{l}\text { Deleterious } \\
(-1.050)\end{array}$ \\
\hline p.P175S & c. $523 C>T$ & $\begin{array}{l}\text { Possibly } \\
\text { damaging (1.000) }\end{array}$ & $\begin{array}{l}\text { Damaging } \\
(0.001)\end{array}$ & $\begin{array}{l}\text { Deleterious } \\
(-1.040)\end{array}$ \\
\hline p.H220P & c.659A $>C$ & $\begin{array}{l}\text { Benign } \\
(0.000)\end{array}$ & $\begin{array}{l}\text { Damaging } \\
(0.004)\end{array}$ & $\begin{array}{l}\text { Tolerated } \\
(2.100)\end{array}$ \\
\hline
\end{tabular}

genotypes for $\mathrm{BH} 4$ responsiveness is of great interest for PKU patients in China.

\section{Responsive Mutations}

Among the eight responsive mutations, four mutations (p.R241C, p.F331S, p.S349A, and p.R408Q) have previously been defined as BH4-responsive mutations. Similar to our findings on the BH4 response of p.R241C, Shintaku et al. (21) observed regularity in p.R241C regardless of the second mutation in the genotype. The second highest frequency responsive mutation, p.R408Q, was less consistently responsive. One out of seven patients was a nonresponder, having the genotype p.[R408Q];[R413P].

Two known mutations (p.A322T and p.Q419R) and two novel mutations (p.L98V, IVS3-2A $>$ T) were first reported as responsive. Three missense mutations (p.L98V, p.A322T and p.Q419R) in the functionally heterozygous genotypes resulted in mild hyperphenylalaninemia/mPKU and responded to $\mathrm{BH} 4$. The splicing mutation IVS3-2A $>\mathrm{T}$ associated with $\mathrm{CPKU}$ was responsive to $\mathrm{BH} 4$, which may be affected by dietary fluctuations during $\mathrm{BH} 4$ loading test, resulting in incorrect assignment. However, some splicing mutations (e.g., IVS4-5C>G and IVS10 $-3 \mathrm{C}>\mathrm{T}$ ) were repeatedly observed to be responsive $(11,22)$, so the $\mathrm{BH} 4$-responsive mechanism of these splicing mutations may be explained by generating a transcript encoding an enzyme with some activity. Therefore, further studies are needed to clarify the residual activities and $\mathrm{BH} 4$-responsive mechanisms of these splicing mutations.

However, four mutations (p.R53H, p.I65T, p.R158Q, and p.R261Q) previously defined as $\mathrm{BH} 4$ responsive were unresponsive to $\mathrm{BH} 4$. Three mutations, including p.I65T $(23,24)$, p.R158Q (12,25), and p.R261Q $(12,23)$, have been repeatedly reported with high rates of discordant $\mathrm{BH} 4$ responsiveness. Moreover, Trefz found mutations with a highly inconsistent rate of $\mathrm{BH} 4$-responsiveness in the regulatory domain (25), where p. $\mathrm{R} 53 \mathrm{H}$ and p.I65T are located. The BH4-responsiveness of these mutations may be more easily affected by interallelic complementation in the genotype or other nongenetic factors (15). Therefore, they cannot be regarded as dominant in the estimation of $\mathrm{BH} 4$ responsiveness. 
Table 3. Genotypes and association with $\mathrm{BH} 4$ responsiveness in 147 patients with two mutations

\begin{tabular}{|c|c|c|c|c|c|c|}
\hline Genotype & Protein variant & PRA (\%) ${ }^{\mathrm{a}}$ & $\begin{array}{l}\text { Data on } \mathrm{BH} 4- \\
\text { responsiveness } \\
\text { of genotype }^{\mathrm{b}}\end{array}$ & \multicolumn{3}{|c|}{$\begin{array}{c}\text { Number of patients } \\
\text { (biochemical phenotype) }^{c}\end{array}$} \\
\hline \multicolumn{7}{|l|}{ Homozygous } \\
\hline c. $[611 A>G] ;[611 A>G]$ & p. $[E X 6-96 A>G] ;[E X 6-96 A>G]$ & Null & NC & & $1 \mathrm{mPKU}+1 \mathrm{cPKU}$ & 2 \\
\hline c. $[498 \mathrm{C}>\mathrm{G}] ;[498 \mathrm{C}>\mathrm{G}]$ & p. $\left[\mathrm{Y} 166^{*}\right] ;\left[\mathrm{Y} 166^{*}\right]$ & Null & NC & & $2 \mathrm{cPKU}$ & 2 \\
\hline c. $[1068 \mathrm{C}>\mathrm{A}] ;[1068 \mathrm{C}>\mathrm{A}]$ & p.[Y356*];[Y356*] & Null & NC & & $1 \mathrm{CPKU}$ & 1 \\
\hline c. $[1223 G>A] ;[1223 G>A]$ & p.[R408Q];[R408Q] & 46 & $\mathrm{R}$ & $1 \mathrm{mPKU}$ & & 1 \\
\hline c. $[1238 G>C] ;[1238 G>C]$ & p.[R413P];[R413P] & 35 & NC & & $2 \mathrm{cPKU}$ & 2 \\
\hline \multicolumn{7}{|l|}{ Heterozygous } \\
\hline c.[206_208delCTT];[770G>T] & p.[P69_S70delinsP];[G257V] & Null+Null & ND & & $1 \mathrm{cPKU}$ & 1 \\
\hline c. [331C>T];[1068C>A] & p.[R111*];[Y356*] & Null+Null & NC & & $1 \mathrm{CPKU}$ & 1 \\
\hline c.[331C>T];[1197A>T] & p.[R111*];[V399V] & Null+Null & NC & & $2 \mathrm{CPKU}$ & 2 \\
\hline c. $[442-1 \mathrm{G}>\mathrm{A}] ;[526 \mathrm{C}>\mathrm{T}]$ & p.[(?)];[R176*] & Null+Null & ND & & $1 \mathrm{CPKU}$ & 1 \\
\hline c. $[442-1 \mathrm{G}>\mathrm{A}] ;[611 \mathrm{~A}>\mathrm{G}]$ & p.[(?)];[EX6-96A>G] & Null+Null & NC & & $1 \mathrm{CPKU}$ & 1 \\
\hline c. $[442-1 \mathrm{G}>\mathrm{A}] ;[799 \mathrm{C}>\mathrm{T}]$ & p.[(?)];[Q267*] & Null+Null & ND & & $1 \mathrm{CPKU}$ & 1 \\
\hline c. $[442-1 \mathrm{G}>\mathrm{A}] ;[1068 \mathrm{C}>\mathrm{A}]$ & p.[(?)];[Y356*] & Null+Null & NC & & $1 \mathrm{CPKU}$ & 1 \\
\hline c. $[442-1 \mathrm{G}>\mathrm{A}] ;[1197 \mathrm{~A}>\mathrm{T}]$ & p.[(?)];[V399V] & Null+Null & NC & & $2 \mathrm{CPKU}$ & 2 \\
\hline c. $[472 \mathrm{C}>\mathrm{T}] ;[611 \mathrm{~A}>\mathrm{G}]$ & p.[R158W];[EX6-96A>G] & Null+Null & ND & & $1 \mathrm{CPKU}$ & 1 \\
\hline c. [498C >G];[722delG] & p.[Y166*];[R241Pfs*100] & Null+Null & ND & & $1 \mathrm{CPKU}$ & 1 \\
\hline c. $[526 \mathrm{C}>\mathrm{T}] ;[1068 \mathrm{C}>\mathrm{A}]$ & p. $\left[\mathrm{R} 176^{*}\right] ;\left[\mathrm{Y} 356^{*}\right]$ & Null+Null & ND & & $1 \mathrm{CPKU}$ & 1 \\
\hline c. $[611 \mathrm{~A}>\mathrm{G}] ;[764 \mathrm{~T}>\mathrm{C}]$ & p.[EX6-96A>G];[L255S] & Null+Null & ND & & $1 \mathrm{CPKU}$ & 1 \\
\hline c.[440C>T];[1215_1219delAATAC] & p.[P147L];[1406Sfs*17] & $?+$ Null & ND & & $1 \mathrm{mPKU}$ & 1 \\
\hline c. $[460 \mathrm{~T}>\mathrm{C}] ;[331 \mathrm{C}>\mathrm{T}]$ & p. $[\mathrm{Y} 154 \mathrm{H}] ;\left[\mathrm{R} 111^{*}\right]$ & $?+$ Null & ND & & $1 \mathrm{cPKU}$ & 1 \\
\hline c. $[473 G>A] ;[331 C>T]$ & p.[R158Q];[R111*] & 10+Null & ND & & $1 \mathrm{CPKU}$ & 1 \\
\hline c. $[721 \mathrm{C}>\mathrm{T}] ;[331 \mathrm{C}>\mathrm{T}]$ & p.[R241C];[R111*] & $25+$ Null & $\mathrm{R} / \mathrm{NR}$ & $1 \mathrm{MHP}$ & & 1 \\
\hline c. $[721 \mathrm{C}>\mathrm{T}] ;[[442-1 \mathrm{G}>\mathrm{A}]$ & p.[R241C];[(?)] & $25+$ Null & $\mathrm{R}$ & $2 \mathrm{mPKU}$ & & 2 \\
\hline c. $[721 \mathrm{C}>\mathrm{T}] ;[478 \mathrm{C}>\mathrm{T}]$ & p.[R241C];[Q160*] & 25+Null & ND & $1 \mathrm{MHP}$ & & 1 \\
\hline c. $[721 \mathrm{C}>\mathrm{T}] ;[498 \mathrm{C}>\mathrm{G}]$ & p.[R241C];[Y166*] & $25+$ Null & NC & $1 \mathrm{mPKU}$ & & 1 \\
\hline c. $[721 \mathrm{C}>\mathrm{T}] ;[611 \mathrm{~A}>\mathrm{G}]$ & p.[R241C];[EX6-96A>G] & $25+$ Null & NC & $1 \mathrm{CPKU}+1 \mathrm{MHP}$ & & 2 \\
\hline c. $[721 \mathrm{C}>\mathrm{T}] ;[1068 \mathrm{C}>\mathrm{A}]$ & p.[R241C];[Y356*] & $25+$ Null & ND & $1 \mathrm{MHP}+1 \mathrm{mPKU}$ & & 2 \\
\hline c. $[721 \mathrm{C}>\mathrm{T}] ;[1197 \mathrm{~A}>\mathrm{T}]$ & p.[R241C];[V399V] & $25+$ Null & NC & $1 \mathrm{mPKU}$ & & 1 \\
\hline c.[728G>A];[30_31insC] & p.[R243Q];[R13Qfs*5] & 14+Null & ND & & $1 \mathrm{CPKU}$ & 1 \\
\hline c.[728G>A];[206_208delCTT] & p.[R243Q];[P69_S70delinsP] & 14+Null & ND & & $1 \mathrm{mPKU}$ & 1 \\
\hline c. $[728 \mathrm{G}>\mathrm{A}] ;[331 \mathrm{C}>\mathrm{T}]$ & p.[R243Q];[R111*] & 14+Null & NC & & $2 \mathrm{CPKU}$ & 2 \\
\hline c. $[728 \mathrm{G}>\mathrm{A}] ;[442-1 \mathrm{G}>\mathrm{A}]$ & p.[R243Q];[(?)] & 14+Null & NC & & $3 c P K U$ & 3 \\
\hline c. $[728 \mathrm{G}>\mathrm{A}] ;[498 \mathrm{C}>\mathrm{G}]$ & p.[R243Q];[Y166*] & 14+Null & NC & & $1 \mathrm{cPKU}$ & 1 \\
\hline c. $[728 \mathrm{G}>\mathrm{A}] ;[526 \mathrm{C}>\mathrm{T}]$ & p.[R243Q];[R176*] & 14+Null & NC & & $1 \mathrm{cPKU}$ & 1 \\
\hline c. $[728 \mathrm{G}>\mathrm{A}] ;[611 \mathrm{~A}>\mathrm{G}]$ & p.[R243Q];[EX6-96A>G] & 14+Null & NC & & $1 \mathrm{mPKU}+5 \mathrm{cPKU}$ & 6 \\
\hline c. $[728 \mathrm{G}>\mathrm{A}] ;[707-1 \mathrm{G}>\mathrm{A}]$ & p.[R243Q];[(?)] & 14+Null & NC & & $2 \mathrm{cPKU}$ & 2 \\
\hline c.[728G>A];[722delG] & p.[R243Q];[R241Pfs*100] & 14+Null & NC & & $1 \mathrm{mPKU}+2 \mathrm{cPKU}$ & 3 \\
\hline c. $[728 \mathrm{G}>\mathrm{A}] ;[781 \mathrm{C}>\mathrm{T}]$ & p.[R243Q];[R261*] & 14+Null & NR & & $1 \mathrm{cPKU}$ & 1 \\
\hline c.[728G>A];[833C>T] & p.[R243Q];[T278I] & 14+Null & NC & & $1 \mathrm{CPKU}$ & 1 \\
\hline c. $[728 \mathrm{G}>\mathrm{A}] ;[1068 \mathrm{C}>\mathrm{A}]$ & p.[R243Q];[Y356*] & 14+Null & NC & & $2 \mathrm{CPKU}$ & 2 \\
\hline
\end{tabular}




\section{Articles | Taoet al.}

Table 3. Continued

\begin{tabular}{|c|c|c|c|c|c|c|}
\hline \multirow[b]{2}{*}{ Genotype } & \multirow[b]{2}{*}{ Protein variant } & \multirow[b]{2}{*}{ PRA (\%) ${ }^{a}$} & \multirow[b]{2}{*}{$\begin{array}{l}\text { Data on } \mathrm{BH} 4- \\
\text { responsiveness } \\
\text { of genotype }^{\mathrm{b}}\end{array}$} & \multicolumn{3}{|c|}{$\begin{array}{c}\text { Number of patients } \\
\text { (biochemical phenotype }^{c}\end{array}$} \\
\hline & & & & $\begin{array}{l}\text { Number of } \\
\text { responsive } \\
\text { patients }\end{array}$ & $\begin{array}{l}\text { Number of } \\
\text { unresponsive } \\
\text { patients }\end{array}$ & $\begin{array}{l}\text { Total of } \\
\text { patients }\end{array}$ \\
\hline c. $[728 \mathrm{G}>\mathrm{A}] ;[1197 \mathrm{~A}>\mathrm{T}]$ & p.[R243Q];[V399V] & 14+Null & $\mathrm{NC}$ & & $1 \mathrm{mPKU}+2 \mathrm{cPKU}$ & 3 \\
\hline c. [739G $>C] ;[442-1 \mathrm{G}>\mathrm{A}]$ & p.[G247R];[(?)] & $?+$ Null & ND & & $1 \mathrm{mPKU}$ & 1 \\
\hline c. $[740 \mathrm{G}>\mathrm{T}] ;[1068 \mathrm{C}>\mathrm{A}]$ & p.[G247V];[Y356*] & $4+$ Null & ND & & $1 c \mathrm{PKU}$ & 1 \\
\hline c.[755G>A];[331C>T] & p.[R252Q];[R111*] & $3+$ Null & NC & & $1 \mathrm{cPKU}$ & 1 \\
\hline c. $[782 \mathrm{G}>\mathrm{A}] ;[498 \mathrm{C}>\mathrm{G}]$ & p.[R261Q];[Y166*] & $44+$ Null & ND & & $1 \mathrm{cPKU}$ & 1 \\
\hline c. $[782 \mathrm{G}>\mathrm{A}] ;[611 \mathrm{~A}>\mathrm{G}]$ & p.[R261Q];[EX6-96A>G] & 44+Null & ND & & $2 \mathrm{cPKU}$ & 2 \\
\hline c.[782G>A];[795C>A] & p.[R261Q];[C265*] & $44+$ Null & ND & & $1 \mathrm{mPKU}$ & 1 \\
\hline c. $[782 \mathrm{G}>\mathrm{A}] ;[1197 \mathrm{~A}>\mathrm{T}]$ & p.[R261Q];[V399V] & $44+$ Null & ND & & $1 \mathrm{cPKU}$ & 1 \\
\hline c. $[875 \mathrm{C}>\mathrm{T}] ;[913-7 \mathrm{~A}>\mathrm{G}]$ & p.[P292L];[(?)] & $?+$ Null & ND & & $1 \mathrm{mPKU}$ & 1 \\
\hline c.[920G>A];[331C>T] & p.[G307D];[R111*] & $?+$ Null & ND & & $1 \mathrm{mPKU}$ & 1 \\
\hline c. [964G>A];[331C>T] & p.[A322T];[R111*] & $?+$ Null & ND & $1 \mathrm{MHP}$ & & 1 \\
\hline c. $[971 \mathrm{~T}>\mathrm{A}] ;[331 \mathrm{C}>\mathrm{T}]$ & p.[I324N];[R111*] & $?+$ Null & ND & & $1 \mathrm{cPKU}$ & 1 \\
\hline c. $[971 \mathrm{~T}>\mathrm{A}] ;[509+1 \mathrm{G}>\mathrm{A}]$ & p.[I324N];[(?)] & $?+$ Null & ND & & $1 \mathrm{cPKU}$ & 1 \\
\hline c.[992T>C];[44_45delTC] & p.[F331S];[S16*] & ?+Null & ND & $1 \mathrm{mPKU}$ & & 1 \\
\hline c. $[1024 G>A] ;[1068 C>A]$ & p.[A342T];[Y356*] & 26+Null & ND & & $1 \mathrm{cPKU}$ & 1 \\
\hline c.[1033G>A];[842C>T] & p.[A345T];[P281L] & $?+$ Null & ND & & $1 \mathrm{mPKU}$ & 1 \\
\hline c.[1045T>G];[611A>G] & p.[S349A];[EX6-96A>G] & ?+Null & ND & $1 \mathrm{mPKU}$ & & 1 \\
\hline c. $[1223 \mathrm{G}>\mathrm{A}] ;[442-1 \mathrm{G}>\mathrm{A}]$ & p.[R408Q];[(?)] & $46+$ Null & ND & $1 \mathrm{mPKU}$ & & 1 \\
\hline c.[1223G>A];[722delG] & p.[R408Q];[R241Pfs*100] & $46+$ Null & ND & $1 \mathrm{CPKU}$ & & 1 \\
\hline c.[1223G>A];[770G>T] & p.[R408Q];[G257V] & $46+$ Null & ND & $1 \mathrm{mPKU}$ & & 1 \\
\hline c.[1238G>C];[331C>T] & p.[R413P];[R111*] & $35+$ Null & NC & & $1 \mathrm{mPKU}$ & 1 \\
\hline c.[1238G>C];[498C>G] & p.[R413P];[Y166*] & $35+$ Null & $\mathrm{NC}$ & & $1 \mathrm{CPKU}$ & 1 \\
\hline c.[1238G>C];[611A>G] & p.[R413P];[EX6-96A>G] & $35+$ Null & $\mathrm{NC}$ & & $1 \mathrm{cPKU}$ & 1 \\
\hline c.[1238G >C];[764T>C] & p.[R413P];[L255S] & $35+$ Null & ND & & $1 \mathrm{cPKU}$ & 1 \\
\hline c. $[1238 G>C] ;[1068 C>A]$ & p.[R413P];[Y356*] & $35+$ Null & NC & $1 \mathrm{cPKU}$ & $2 \mathrm{cPKU}$ & 3 \\
\hline c. $[1238 G>C] ;[1197 A>T]$ & p.[R413P];[V399V] & $35+$ Null & $\mathrm{NC}$ & & $2 \mathrm{cPKU}$ & 2 \\
\hline c. $[1256 \mathrm{~A}>\mathrm{G}] ;[1068 \mathrm{C}>\mathrm{A}]$ & p.[Q419R];[Y356*] & $71+$ Null & ND & $1 \mathrm{MHP}$ & & 1 \\
\hline c. $[1301 \mathrm{C}>\mathrm{A}] ;[611 \mathrm{~A}>\mathrm{G}]$ & p.[A434D];[EX6-96A>G] & $3+$ Null & ND & $1 \mathrm{MHP}$ & $1 \mathrm{mPKU}$ & 2 \\
\hline c.[143T>C];[739G >C] & p.[L48S];[G247R] & $39+?$ & ND & $1 \mathrm{mPKU}$ & & 1 \\
\hline c. $[158 \mathrm{G}>\mathrm{A}] ;[\mathrm{728G}>\mathrm{A}]$ & p.[R53H];[R243Q] & $79+14$ & $\mathrm{R}$ & & $1 \mathrm{MHP}$ & 1 \\
\hline c.[194T>C];[728G>A] & p.[165T];[R243Q] & $33+14$ & $\mathrm{R}$ & & $1 \mathrm{CPKU}$ & 1 \\
\hline c. $[224 \mathrm{~A}>\mathrm{T}] ;[1238 \mathrm{G}>\mathrm{C}]$ & p.[D75V];[R413P] & $?+35$ & ND & & $1 \mathrm{cPKU}$ & 1 \\
\hline c.[466G $>C] ;[659 A>C]$ & p.[A156P];[H220P] & $?+?$ & ND & & $1 \mathrm{cPKU}$ & 1 \\
\hline c. $[473 \mathrm{G}>\mathrm{A}] ;[740 \mathrm{G}>\mathrm{T}]$ & p.[R158Q];[G247V] & $10+4$ & ND & & $1 \mathrm{cPKU}$ & 1 \\
\hline c. $[516 \mathrm{G}>\mathrm{T}] ;[728 \mathrm{G}>\mathrm{A}]$ & p.[Q172H];[R243Q] & $?+14$ & ND & $1 \mathrm{MHP}$ & & 1 \\
\hline c. $[523 \mathrm{C}>\mathrm{T}] ;[1238 \mathrm{G}>\mathrm{C}]$ & p.[P175S];[R413P] & $?+35$ & ND & & $1 \mathrm{cPKU}$ & 1 \\
\hline c. [563G $>A] ;[728 G>A]$ & p.[G188D];[R243Q] & $?+14$ & ND & & $1 \mathrm{CPKU}$ & 1 \\
\hline c. $[617 A>G] ;[721 C>T]$ & p.[Y206C];[R241C] & $?+25$ & ND & $1 \mathrm{mPKU}$ & & 1 \\
\hline c. $[699 \mathrm{C}>\mathrm{G}] ;[740 \mathrm{G}>\mathrm{T}]$ & p.[F233L];[G247V] & $?+4$ & ND & $1 \mathrm{mPKU}$ & & 1 \\
\hline c.[721C>T];[728G>A] & p.[R241C];[R243Q] & $25+14$ & $\mathrm{R}$ & $2 \mathrm{mPKU}$ & & 2 \\
\hline c. $[721 C>T] ;[1316-2 A>C]$ & p.[R241C];[(?)] & $25+?$ & ND & $1 \mathrm{mPKU}$ & & 1 \\
\hline c. $[728 \mathrm{G}>\mathrm{A}] ;[739 \mathrm{G}>\mathrm{C}]$ & p.[R243Q];[G247R] & $14+?$ & ND & & $1 \mathrm{mPKU}$ & 1 \\
\hline c. $[728 \mathrm{G}>\mathrm{A}] ;[1223 \mathrm{G}>\mathrm{A}]$ & p.[R243Q];[R408Q] & $46+14$ & NC & $2 \mathrm{mPKU}$ & & 2 \\
\hline c. $[728 \mathrm{G}>\mathrm{A}] ;[1238 \mathrm{G}>\mathrm{C}]$ & p.[R243Q];[R413P] & $14+35$ & NC & & $8 c P K U$ & 8 \\
\hline c. $[728 \mathrm{G}>\mathrm{A}] ;[1301 \mathrm{C}>\mathrm{A}]$ & p.[R243Q];[A434D] & $14+3$ & NC & & $1 \mathrm{mPKU}$ & 1 \\
\hline c. [739G >C];[1238G>C] & p.[G247R];[R413P] & $?+35$ & NC & & $1 \mathrm{cPKU}$ & 1 \\
\hline c.[740G>T];[782G>A] & p.[G247V];[R261Q] & $4+44$ & ND & $1 \mathrm{mPKU}$ & & 1 \\
\hline c. $[755 \mathrm{G}>\mathrm{A}] ;[1031 \mathrm{G}>\mathrm{A}]$ & p.[R252Q];[G344D] & $3+?$ & ND & & $1 \mathrm{cPKU}$ & 1 \\
\hline c. [827T>A];[1238G>C] & p.[M276K];[R413P] & $?+35$ & ND & $1 \mathrm{MHP}$ & & 1 \\
\hline c. $[1099 \mathrm{C}>\mathrm{G}] ;[1174 \mathrm{~T}>\mathrm{A}]$ & p.[L367V];[F392I] & $?+98$ & ND & $1 \mathrm{MHP}$ & & 1 \\
\hline c. $[1223 G>A] ;[1238 G>C]$ & p.[R408Q];[R413P] & $46+35$ & NC & & $1 \mathrm{cPKU}$ & 1 \\
\hline c. $[1223 G>A] ;[1252 A>C]$ & p.[R408Q];[T418P] & $46+?$ & ND & $1 \mathrm{MHP}$ & & 1 \\
\hline
\end{tabular}

aData obtained from the BIOPKU database (15). Null mutations contained nonsense, frame-shift, splicing mutations(only known mutation) and those missense mutations with less than $3 \%$ enzyme activity in vitro? - unknown PRA. 'Data obtained from the BIOPKU database (15). ND, no data on genotype; R, responder; NR, nonresponder; NC, not clear about $\mathrm{BH} 4$-responsiveness although having data on genotype. ${ }^{\mathrm{C}} \mathrm{PKU}$, classic PKU; mPKU, mild PKU; MHP, mild hyperphenylalaninemia. Patients were assigned to three biochemical phenotype categories according to their pretreatment plasma Phe levels. 
Table 4. Genotypes with additional mutations in cis and $\mathrm{BH} 4$ responsiveness in six patients

\begin{tabular}{|c|c|c|c|c|}
\hline Patient and parents & Biochemical phenotype $^{b}$ & Genotype & Protein variant & Phe red. $24 \mathrm{~h}(\%)$ \\
\hline $1(\mathrm{SJZ})^{\mathrm{a}}$ & CPKU & c. $[331 \mathrm{C}>\mathrm{T}] ;[158 \mathrm{G}>\mathrm{A} ; 842+2 \mathrm{~T}>\mathrm{A}]$ & p.[R111*];[R53H;(?)] & 8.06 \\
\hline Father & - & c. $[158 \mathrm{G}>\mathrm{A}] ;[842+2 \mathrm{~T}>\mathrm{A}]$ & p.[R53H];[(?)] & - \\
\hline $2(S X)^{a}$ & cPKU & c. $[728 \mathrm{G}>\mathrm{A}] ;[158 \mathrm{G}>\mathrm{A} ; 842+2 \mathrm{~T}>\mathrm{A}]$ & p.[R243Q];[R53H; (?)] & 4.66 \\
\hline Father & - & c. $[158 \mathrm{G}>\mathrm{A}] ;[842+2 \mathrm{~T}>\mathrm{A}]$ & p.[R53H];[(?)] & - \\
\hline $3(C Z)^{a}$ & $\mathrm{mPKU}$ & c. $[650 \mathrm{G}>\mathrm{A}] ;[158 \mathrm{G}>\mathrm{A} ; 842+2 \mathrm{~T}>\mathrm{A}]$ & p.[C217Y];[R53H; (?)] & 3.85 \\
\hline Father & - & c. $[650 G>A] ;[=]$ & p.[C217Y];[=] & - \\
\hline Mother & - & c.[158G>A];[?] & p.[R53H];[(?)] & - \\
\hline $4(\mathrm{JL})^{\mathrm{a}}$ & $\mathrm{mPKU}$ & c. $[1197 \mathrm{~A}>\mathrm{T}] ;[158 \mathrm{G}>\mathrm{A} ; 842+2 \mathrm{~T}>\mathrm{A}]$ & p.[V399V];[R53H; (?)] & -1.88 \\
\hline Father & - & c. $[1197 A>T] ;[=]$ & p.[V399V];[=] & - \\
\hline Mother & - & c. $[1197 \mathrm{~A}>\mathrm{T}] ;[=]$ & p.[V399V];[=] & - \\
\hline $6(S X)^{a}$ & $\mathrm{mPKU}$ & c. $[158 \mathrm{G}>\mathrm{A} ; 842+2 \mathrm{~T}>\mathrm{A}] ;[158 \mathrm{G}>\mathrm{A} ; 842+2 \mathrm{~T}>\mathrm{A}]$ & p.[R53H; (?)];[R53H; (?)] & -9.96 \\
\hline Father & - & c. $[158 \mathrm{G}>\mathrm{A}] ;[842+2 \mathrm{~T}>\mathrm{A}]$ & p.[R53H];[(?)] & - \\
\hline Mother & - & c. $[158 \mathrm{G}>\mathrm{A}] ;[842+2 \mathrm{~T}>\mathrm{A}]$ & p.[R53H];[(?)] & - \\
\hline
\end{tabular}

aThese patients were from four different cities: three from Shanxi (SX), one from Cangzhou (CZ), one from Jilin (JL), and one from Shijiazhuang (SJZ). BH4-responsive mutations in bold were obtained from the BIOPKU database (15). ${ }^{b} \mathrm{CPKU}$, classic PKU; mPKU, mild PKU; MHP, mild hyperphenylalaninemia.

\section{Genotype and Its Correlation With BH4 Responsiveness}

Because most patients are compound heterozygotes, the complete genotype is a precondition for accurately predicting $\mathrm{BH} 4$ responsiveness. We found that patients with genotypes that lack residual "PAH activity" showed the highest degree of concordance with unresponsiveness, as previously reported (9). Residual PAH activity of at least $12.5 \%$ was required for responsive genotype, similar to the previous finding that a full genotype characterized by a residual activity of at least $15.5 \%$ was needed for response (11). However, the same genotypes with disparate $\mathrm{BH} 4$ responsiveness and genotypes with high residual PAH activities yet unresponsiveness were observed. Thus, the association between genotypes and $\mathrm{BH} 4$ responsiveness in our patients is uncertain, supporting the previous findings that genotype is not an absolute predictor of $\mathrm{BH} 4$ responsiveness $(9,10,25)$. These discrepancies were mostly due to the difference of the residual PAH activity in vitro and in vivo. The residual $\mathrm{PAH}$ activity in vitro may be subject to the regulating function of gene variances in the introns of the $P A H$ gene (26) and interallelic complementation (27). Accordingly, the residual PAH activity in vivo may be also affected by the genetic factors and the other regulating factors (e.g., $\mathrm{BH} 4$ pharmacokinetics, protein catabolic rate, and Phe intake during the test) in the interindividual variances (28-30).

As a general rule, the genotype consisted of two mutations in PKU patients. The reason for only one causative mutation found in 12 patients may be a large deletion or a mutation in the intronic sequences far from the exons or in the $5^{\prime}$ and $3^{\prime}$ untranslated regions, yet to be detected, at the PAH locus. However, six patients with atypical genotypes with three or even four mutations were observed. Such unusual situations were recently documented by other researchers $(9,12,31)$, but few studies have investigated the $\mathrm{BH} 4$ responsiveness of genotypes with more than two mutations. Our findings agreed with other studies, that patients with the genotype carrying one or two defined responsive mutations and displaying a substantial residual activity did not respond to $\mathrm{BH} 4$. One patient was carrying two responsive mutations in cis (p.R241C and p.V245A) in combination with one mutation (p.R408W) (12), and another patient was harboring two mutations in cis (p.Y277C and p.V245A) in combination with one mutation (IVS12+1G>A) (31). In addition to interallelic complementation and many regulating elements, part of these anomalies may be explained by a specific mutation that is dominant in the genotype-based classification of $\mathrm{BH} 4$ responsiveness.

As stated above, the strength of our study is providing clinicians with more genotypic information on $\mathrm{BH} 4$ responsiveness before the $\mathrm{BH} 4$ loading test. Our results highlight the significance of screening the complete $P A H$ genotype to increase the accuracy of genotype-based prediction of $\mathrm{BH} 4$ responsiveness. However, some limitations should be noted. First, the $\mathrm{BH} 4$ responsiveness of genotypes may be misclassified when only based on the results of the 24-h loading test. Some of the patients who benefited from $\mathrm{BH} 4$ therapy responded until $48 \mathrm{~h}$ after a $\mathrm{BH} 4$ trial (32), whereas initial responders were negative to BH4 after a long-term treatment (3). Second, the effect of a mutant genotype, especially for a genotype harboring one novel mutation or having more than two mutations, on protein structure and molecular function requires further study. 
In conclusion, genotype-based predictions of $\mathrm{BH} 4$ responsiveness are only designed to identify patients who have a high probability of responding to $\mathrm{BH} 4$. Potential responders will be tested with a long-term $\mathrm{BH} 4$ challenge.

\section{METHODS}

\section{Patient Enrollment}

From August 2012 to August 2013, we recruited 165 PKU patients (91 males and 74 females) from 21 newborn screening centers in China. All patients fulfilled the diagnostic criteria of PKU, with a blood phenylalanine concentration $>2 \mathrm{mg} / \mathrm{dl}$. BH4 deficiency was excluded by analysis of urinary pterins and dihydropteridine reductase activity in erythrocytes. Demographic data (basic information of the patient and family history of PKU) and clinical examination results (physical examinations and laboratory tests) were collected by a detailed and structured questionnaire. The patient ages ranged from $27 \mathrm{~d}$ to $13 \mathrm{y}$ old (a median age of $3 \mathrm{y}$ ). These patients had undergone the same $\mathrm{BH} 4$ loading test/Phe+BH4 loading test in clinic. A single dose of $20 \mathrm{mg} / \mathrm{kg}$ exogenous $\mathrm{BH} 4$ (sapropterin dihydrochloride, BioMarin Pharmaceutical, CA) was used for the 24-h BH4 loading test performed in these patients, whereas $100 \mathrm{mg} / \mathrm{kg}$ Phe (Phenylalanine, Chinese and Western three-dimensional Pharmaceutical, Shanghai, China) was administered $3 \mathrm{~h}$ before the BH4 loading test in patients with $<10 \mathrm{mg} / \mathrm{dl}$ blood Phe level. During the test, the patients maintained their usual diets without restricting Phe intake. Plasma Phe levels were taken at $T=0,2,4,8$, and $24 \mathrm{~h}$. A patient is defined as a BH4-responder when there is a reduction of at least $30 \%$ in the blood phenylalanine concentration after the 24-h BH4 challenge (7).

This research was approved by the Ethics Committee of Sichuan University (No.2014018), and informed consent was obtained from the parents of the patients for the molecular study.

\section{DNA Extraction}

A blood sample (4 $\mathrm{ml})$ was collected, in ethylene diamine tetraacetic acid, from the patients and their parents by venipuncture. Genomic DNA was extracted using a DNA extraction kit (Qiagen, Hilden, Germany).

\section{Genotype Analysis With NGS}

A series of unique primers (BoJin Biotechnology, Shanghai, China) was designed to amplify 13 exons and surrounding introns in patients, covering $200 \mathrm{bp}$ upstream of the exon and $200 \mathrm{bp}$ downstream of the exon of the $P A H$ gene. A pair of primers was used to amplify approximately $150 \mathrm{bp}$ DNA of the human $\beta$-globin (HBB) gene (accession number AY260740) as the internal quality control for identifying the false negatives caused by inadequate DNA or failed polymerase chain reaction (PCR).

To improve the throughput of the assay, the PCR primers not only amplify the target DNA but also provide a unique primer index for each of the 96 samples in each plate (multiple index PCR). The result is 96 sets of 10-bp nucleic acid tags at the $5^{\prime}$ end of each $P A H$ and $H B B$ primer (BoJin Biotechnology). The second index for each sample is the 8-bp nucleic acid tags in the library adapter sequence identifying each sample as coming from a specific 96-well plate. This index was attached to amplicons of the samples through the adapter preparation process. Using this "double index system," hundreds of samples can be mixed together and detected in one sequencing chip at the same time.

PCR was conducted in a GeneAmp PCR system 9700 (Applied Biosystems, Foster City, CA), with a cycling protocol consisting of denaturation at $94^{\circ} \mathrm{C}$ for $30 \mathrm{~s}, 56^{\circ} \mathrm{C}$ for $30 \mathrm{~s}$, and $72^{\circ} \mathrm{C}$ for $1 \mathrm{~min}$. After 35 cycles, gel electrophoresis was performed to check the quality of the amplified DNA. Only qualified DNA was allowed for the library preparation. PCR amplification products were prepared for DNA pooling, and diverse adapters were added to the amplification products. After the quantitative concentration satisfied the requirement of the library preparation, gene mutations were sequenced by an Illumina Hiseq 2000 sequencing instrument (Illumina, San Diego, CA).

After sequencing the samples, the raw sequence data were analyzed using in-house software. First, all sequence data were traced back to the specimens according to the sequences of primer indices and adapter indices. Second, the amplicon sequences of every sample were aligned with the standard reference for $P A H$ sequences (NC_000012.11) in GenBank; SNPs were found in the target areas and relevant information was noted. The mutation nomenclature is in accordance with the guidelines from the Human Genome Variation Society (33).

\section{Genotypic Validation}

All mutations identified in patients were confirmed by analyzing parental DNA. When mutation loci were detected in patients, the same locus of the parental sample was amplified by PCR and analyzed by Sanger automated sequencing.

For newly detected mutations not present in the PAH database (McGill University, Montreal, Canada) (34) and in the BIOPKU database (University of Zurich, Zurich, Switzerland) (15), we randomly selected 200 healthy individuals and referred to the Exome Variant Server (35) to rule out the possibility of polymorphisms. Furthermore, the possible pathologic effect of missense mutations was predicted using PolyPhen-2, SIFT Blink, and SNPs3D (36).

\section{Prediction of Responsiveness to $\mathrm{BH} 4$}

The predicted residual PAH activity of one mutation in vitro refers to the BIOPKU database. Null mutations contained nonsense, frameshift, and splicing mutations (only known mutations), and the missense mutations had less than $3 \%$ enzyme activity in vitro (36). The assigned $\mathrm{BH} 4$ responsiveness of one mutation is based on its effect in the homozygous form or functionally heterozygous form (one mutation in combination with a null mutation) (10).

\section{ACKNOWLEDGMENTS}

The authors thank all the children and their parents for participating in this study and for their co-operation, as well as the staff of newborn screening centers involved in the project process for recruitment and data collection. J.T. and J.Z. developed the study design, conducted the study, and drafted the manuscript. Z.L. assisted in organizing and collecting the samples. N.L. and H.J. carried out genetic data acquisition and interpretation. X.L. and J.S. analyzed the data. Y.D. and X.J. participated in reviewing, editing, and revising the manuscript. All authors read and approved the final manuscript.

\section{STATEMENT OF FINANCIAL SUPPORT}

This study was supported by the National "Twelfth Five-Year" Plan for Science \&Technology Support of China (grant ID: 2012BAI09B04 and 2014BAI06B01). The authors have no other funding or conflicts of interest to disclose.

\section{REFERENCES}

1. Blau N, van Spronsen FJ, Levy HL. Phenylketonuria. Lancet 2010;376: 1417-27.

2. Shi XT, Cai J, Wang YY, et al. Newborn screening for inborn errors of metabolism in mainland china: 30 years of experience. JIMD Rep 2012;6:79-83.

3. Levy HL, Milanowski A, Chakrapani A, et al.; Sapropterin Research Group. Efficacy of sapropterin dihydrochloride (tetrahydrobiopterin, 6R-BH4) for reduction of phenylalanine concentration in patients with phenylketonuria: a phase III randomised placebo-controlled study. Lancet 2007;370:504-10.

4. Trefz FK, Burton BK, Longo N, et al.; Sapropterin Study Group. Efficacy of sapropterin dihydrochloride in increasing phenylalanine tolerance in children with phenylketonuria: a phase III, randomized, double-blind, placebo-controlled study. J Pediatr 2009;154:700-7.

5. Keil S, Anjema K, van Spronsen FJ, et al. Long-term follow-up and outcome of phenylketonuria patients on sapropterin: a retrospective study. Pediatrics 2013;131:e1881-8.

6. Burlina A, Blau N. Effect of $\mathrm{BH}(4)$ supplementation on phenylalanine tolerance. J Inherit Metab Dis 2009;32:40-5.

7. Fiege B, Blau N. Assessment of tetrahydrobiopterin (BH4) responsiveness in phenylketonuria. J Pediatr 2007;150:627-30.

8. Muntau AC, Röschinger W, Habich $\mathrm{M}$, et al. Tetrahydrobiopterin as an alternative treatment for mild phenylketonuria. $\mathrm{N}$ Engl $\mathrm{J}$ Med 2002;347:2122-32.

9. Dobrowolski SF, Heintz C, Miller T, et al. Molecular genetics and impact of residual in vitro phenylalanine hydroxylase activity on tetrahydrobiopterin 
responsiveness in Turkish PKU population. Mol Genet Metab 2011;102:116-21.

10. Zurflüh MR, Zschocke J, Lindner M, et al. Molecular genetics of tetrahydrobiopterin-responsive phenylalanine hydroxylase deficiency. Hum Mutat 2008;29:167-75.

11. Tansek MZ, Groselj U, Murko S, Kobe H, Lampret BR, Battelino T. Assessment of tetrahydrobiopterin $(\mathrm{BH}(4))$-responsiveness and spontaneous phenylalanine reduction in a phenylalanine hydroxylase deficiency population. Mol Genet Metab 2012;107:37-42.

12. Karacić I, Meili D, Sarnavka V, et al. Genotype-predicted tetrahydrobiopterin (BH4)-responsiveness and molecular genetics in Croatian patients with phenylalanine hydroxylase (PAH) deficiency. Mol Genet Metab 2009;97:165-71.

13. Erlandsen H, Pey AL, Gámez A, et al. Correction of kinetic and stability defects by tetrahydrobiopterin in phenylketonuria patients with certain phenylalanine hydroxylase mutations. Proc Natl Acad Sci USA 2004;101:16903-8.

14. Pey AL, Pérez B, Desviat LR, et al. Mechanisms underlying responsiveness to tetrahydrobiopterin in mild phenylketonuria mutations. Hum Mutat 2004;24:388-99.

15. Blau N, Shen N, Carducci C. Molecular genetics and diagnosis of phenylketonuria: state of the art. Expert Rev Mol Diagn 2014;14:655-71.

16. Song F, Qu YJ, Zhang T, Jin YW, Wang H, Zheng XY. Phenylketonuria mutations in Northern China. Mol Genet Metab 2005;86 Suppl 1:S107-18.

17. Shendure J, Ji H. Next-generation DNA sequencing. Nat Biotechnol 2008;26:1135-45.

18. Trujillano D, Perez B, González J, et al. Accurate molecular diagnosis of phenylketonuria and tetrahydrobiopterin-deficient hyperphenylalaninemias using high-throughput targeted sequencing. Eur J Hum Genet 2014;22:528-34.

19. Gu Y, Lu K, Yang G, et al. Mutation spectrum of six genes in Chinese phenylketonuria patients obtained through next-generation sequencing. PLoS One 2014;9:e94100.

20. Lagler FB, Gersting SW, Zsifkovits C, et al. New insights into tetrahydrobiopterin pharmacodynamics from Pah enu1/2, a mouse model for compound heterozygous tetrahydrobiopterin-responsive phenylalanine hydroxylase deficiency. Biochem Pharmacol 2010;80:1563-71.

21. Shintaku H, Kure S, Ohura T, et al. Long-term treatment and diagnosis of tetrahydrobiopterin-responsive hyperphenylalaninemia with a mutant phenylalanine hydroxylase gene. Pediatr Res 2004;55:425-30.

22. Fiege B, Bonafé L, Ballhausen D, et al. Extended tetrahydrobiopterin loading test in the diagnosis of cofactor-responsive phenylketonuria: a pilot study. Mol Genet Metab 2005;86 Suppl 1:S91-5.

23. Couce ML, Bóveda MD, Fernández-Marmiesse A, et al. Molecular epidemiology and BH4-responsiveness in patients with phenylalanine hydroxylase deficiency from Galicia region of Spain. Gene 2013;521:100-4.

24. Desviat LR, Pérez B, Bèlanger-Quintana A, et al. Tetrahydrobiopterin responsiveness: results of the $\mathrm{BH} 4$ loading test in 31 Spanish PKU patients and correlation with their genotype. Mol Genet Metab 2004;83:157-62.

25. Trefz FK, Scheible D, Götz H, Frauendienst-Egger G. Significance of genotype in tetrahydrobiopterin-responsive phenylketonuria. J Inherit Metab Dis 2009;32:22-6.
26. Stojiljkovic M, Zukic B, Tosic N, et al. Novel transcriptional regulatory element in the phenylalanine hydroxylase gene intron 8. Mol Genet Metab 2010;101:81-3.

27. Leandro J, Nascimento C, de Almeida IT, Leandro P. Co-expression of different subunits of human phenylalanine hydroxylase: evidence of negative interallelic complementation. Biochim Biophys Acta 2006;1762:544-50.

28. Staudigl M, Gersting SW, Danecka MK, et al. The interplay between genotype, metabolic state and cofactor treatment governs phenylalanine hydroxylase function and drug response. Hum Mol Genet 2011;20: 2628-41.

29. Fiege B, Ballhausen D, Kierat L, et al. Plasma tetrahydrobiopterin and its pharmacokinetic following oral administration. Mol Genet Metab 2004;81:45-51.

30. Leuzzi V, Carducci C, Carducci C, et al. The spectrum of phenylalanine variations under tetrahydrobiopterin load in subjects affected by phenylalanine hydroxylase deficiency. J Inherit Metab Dis 2006;29:38-46.

31. Lambruschini N, Perez-Duenas B, Vilaseca MA, et al. The spectrum of phenylalanine variations under tetrahydrobiopterin load in subjects affected by phenylalanine hydroxylase deficiency. Mol Genet Metab 2005;86: S54-S60.

32. Anjema K, van Rijn M, Hofstede FC, et al. Tetrahydrobiopterin responsiveness in phenylketonuria: prediction with the 48-hour loading test and genotype. Orphanet J Rare Dis 2013;8:103.

33. Murad H, Dabboul A, Moassas F, Alasmar D, Al-Achkar W. Mutation spectrum of phenylketonuria in Syrian population: genotype-phenotype correlation. Gene 2013;528:241-7.

34. Hoang L, Byck S, Prevost L, Scriver CR. PAH Mutation Analysis Consortium Database: a database for disease-producing and other allelic variation at the human PAH locus. Nucleic Acids Res 1996;24:127-31.

35. Bueno MA, González-Lamuño D, Delgado-Pecellín C, et al. Molecular epidemiology and genotype-phenotype correlation in phenylketonuria patients from South Spain. J Hum Genet 2013;58:279-84.

36. Wettstein S, Underhaug J, Perez B, et al. Linking genotypes database with locus-specific database and genotype-phenotype correlation in phenylketonuria. Eur J Hum Genet 2015;23:302-9.

(c) (1) () $\odot$ This work is licensed under a Creative Commons Attribution-NonCommercial-NoDerivs 4.0 International License. The images or other third party material in this article are included in the article's Creative Commons license, unless indicated otherwise in the credit line; if the material is not included under the Creative Commons license, users will need to obtain permission from the license holder to reproduce the material. To view a copy of this license, visit http://creativecommons.org/ licenses/by-nc-nd/4.0/ 